\title{
FEASIBILITY STUDIES ON BLACK START CAPABILITY OF DISTRIBUTED ENERGY RESOURCES
}

\author{
Weijie Yan $^{1 *}$, Qiteng Hong ${ }^{1}$, Di Liu ${ }^{1}$, Adam Dyśko ${ }^{1}$ \\ ${ }^{1}$ Department of Electronic and Electrical Engineering, University of Strathclyde, G1 1XW, Glasgow, U. K. \\ *weijie.yan@strath.ac.uk
}

Keywords: BLACK START, DISTRIBUTED GENERATION, FEASIBILITY

\begin{abstract}
Power systems are experiencing massive changes in their generation patterns with increasing penetration of decentralised renewable generation and decommissioning of thermal plants. Such changes in generation patterns will bring significant challenges to the black start arrangement. Conventional black start process relies on large thermal plants connected at the transmission level to energise the system using a "top-down" approach. This may no longer be adequate or applicable in future networks due to the closure of most thermal plants. This paper will investigate the feasibility of using Distributed Energy Resources (DERs) to provide a "bottom-up" black start approach. Compared with the conventional approach, using DERs for black start has potential advantages of reduced restoration time and more flexible recovery procedure. In this paper, a comprehensive review of the existing black start studies utilising DERs will be provided. Additionally, several case studies using a distribution network model containing multiple resources such as wind, micro-hydro generator, and energy storage will be presented to demonstrate the feasibility of providing black start capability by DERs. Furthermore, the associated technical issues that need to be considered to enable such a black start approach will also be discussed, along with feasible solutions and suggestions for further investigation.
\end{abstract}

\section{Introduction}

In recent years, power systems worldwide have seen a massive increase of renewable generation to address the climate change challenges, and this trend is expected to continue in come decades. According to "Future Energy Scenarios" published by National Grid ESO in the U.K. in 2020 [1], in order to meet the net zero carbon emissions target by 2050 , wind and solar, co-located with storage will dominate the future electricity supply under both "Leading the Way" and "Consumer Transformation" scenarios. More specifically, the decentralisation progress requires at least $3 \mathrm{GW}$ of wind and $1.4 \mathrm{GW}$ of solar to be built each year from now until 2050 .

Meanwhile, alongside the 2050 zero carbon target, the British government has put forward the ambitious proposal, the "clean growth strategy", which aims to grow its economy while significantly reducing carbon emissions. It points out that, in order to grow low carbon sources of electricity and deliver a smarter, more flexible, efficient, and resilient energy network, the electricity generation from renewables and nuclear will make up more than $80 \%$ in the future energy generation, and the unabated coal power will be phased out by 2025 [2].

Massive integration of Renewable Energy Sources (RESs) can bring significant challenges to the existing power grid, such as degraded power quality, issues with grid reliability and economic constraints, due to the intermittent and nondispatchable nature of the RESs [3]. From the perspective of power restoration, especially the black start services, it will also re-shape the current fleet. Power system blackout is a High Impact, Low Probability (HILP) event. Although the occurrence rate is low, system blackouts not only can adversely threaten economic development and transport but also severely affect people's daily life. Examples of major blackouts in recent history include 2003 North American blackout, 2006 European blackout, and 2012 Indian blackout [4]. Therefore, it is crucial to develop effective and economic black start strategies in the context of massive integration of RESs so as to minimise the interruption time and economic loss.

"Black start" refers to restoring the power supply to a certain network without relying on external power sources following a complete or partial blackout $[4,5]$. Conventionally, the black start approach relies mainly on large thermal or large hydro power stations connected to the high-voltage transmission network, which is known as "top-down" strategy [6]. The need for the displacement of conventional fossil fuel powered sources with RESs will lead to the decommissioning of many conventional thermal plants that have been used so far as the main black start sources, which could result in the conventional approach no longer applicable or sufficient. However, as mentioned earlier, the continuing changes in the generation mix also provide opportunities to explore alternative black start approaches, one of which, often termed "bottom-up" strategy, focuses on medium-voltage or lowvoltage distribution network with the aid of DERs. Compared to the "top-down" strategy, the "bottom-up" strategy is able to reduce the recovery time and simplify the restoration procedure due to the reduced number of controllable variables including loads, switches, and micro sources [6]. Numerous 
studies have been conducted using the "bottom-up" approach, which are detailed in [3].

The remainder of the paper is organised as follows. Section 2 illustrates the typical technical requirements for black start services and the new requirements as the result of the reduction of the thermal power plants and the integration of the RESs. Section 3 presents the strengths and weaknesses when considering participation of various types of RESs in black start services. In section 4 , case studies based on a distribution network model containing a wind turbine, a mini hydro power plant and an energy storage unit is implemented to investigate the feasibility of DERs to provide black start capability, along with related technical issues and practicable solutions.

\section{Technical Requirements for Black Start}

A DER Black-Start Unit (BSU), which is defined as a microsource possessing the self-startup capability, should meet several basic requirements for black start services: starting up independently from external power supply, energising transmission network, and supplying local loads [7]. Adequate control of voltage and frequency is of major importance. The voltage and frequency must be restricted to a certain range, otherwise, user equipment can be damaged during the restoration process when either voltage or frequency exceeds the acceptable range. In [8], the feasibility of various black start plans is validated by both steady state and dynamic analyses. The steady state analysis includes voltage control and steady-state overvoltage analysis, reactive power absorption capability of BSUs, step-by-step simulation test, robustness verification test, and generation and load matching capability demonstration. In the dynamic analysis, load-frequency control, voltage control, large induction motor starting, motor starting sequence assessment, self-excitation assessment, system stability, and transient overvoltages are investigated.

Additionally, as a result of decommissioning of traditional coal-fired power plants and emergence of RESs, several new attributes need to be considered to guarantee the consistency and the coherence of the black start capability of the existing power grids. As stated in [9], a series of factors need to be considered, such as location (proximity to other stations and other black start stations), restarting ability (start-up time from cold), block loading capability, reactive power capability, connectivity (voltage level and number of circuits), number and size of main and auxiliary starting units, frequency and voltage regulation, inertia, expected availability and reliability of the station, training and testing requirements, and degree of participation in future energy mix. More detailed technical requirements for generator capabilities needed in black start, ancillary services, availability and reliability requirements in addition to the proposed changes to technical specifications can be found in [10].

\section{DERs' Suitability for Black Start}

Generally, the conventional restoration process of the power system is slow due to several factors, such as incomplete and/or inaccurate system data, system vulnerability and deficient regulation capability, and restricted generation capacity to supply critical loads [11]. It is believed that those drawbacks can be mitigated, at least to some extent, by participation of RESs in the black start service.

\subsection{Wind Energy}

Wind energy can be a feasible option considering it is capable of operating at variable speed, controlling real and reactive power, converter rating reduction, rapid ramp-up ability, and multiple start-up capabilities $[12,13]$. However, it also suffers from weather dependence, high intermittence along with low contribution to network inertia, which can negatively impact the frequency and voltage stability of the existing utility grid $[4,10]$. These challenges necessitate the wind energy to be able to possess the frequency and voltage regulation capability, and an external power supply to support start-up (for example, energy storage device) due to the absence of self-starting capability, so as to act an essential role in supporting power system restoration [13].

In the literature review, an offline restoration planning tool was proposed in [14] to tackle the problem of wind variability and uncertainty, where the tool was shown to be able to harness the wind energy in an effective manner, determine optimal wind dispatch decisions while reducing computational burden and restoration time. In [15], a black start scheme using Permanent Magnet Synchronous Generator (PMSG)-based wind farm under the support of diesel generator as an external power supply was proved to be able to energise the outlet transformer of the wind farm, the transmission line, and the adjacent thermal power plant. In [12] and [16], the theory of utilising Doubly-Fed Induction Generator (DFIG) wind turbine-based wind farm with a storage system was modelled and verified its feasibility to involve in black start process after blackouts. In [13], in order to realise a more comprehensive capability of a BSU, for instance, active-reactive power output control of BSU and distribution control of active and reactive power for wind farm, a hybrid BSU consist of combustion gas turbine, Variable Speed Wind Turbine (VSWT)-based wind farm and static synchronous compensator (STATCOM) was simulated in a three-level control model. In [17], an islanded microgrid model including DFIG-based wind turbine, diesel engine driven synchronous generator and energy storage system was tested successfully to achieve frequency control during load step changes.

\subsection{Photovoltaics $(P V)$}

The application of PVs for black start services can also be a viable choice. Similar to wind energy, if controlled properly, the PV can also potentially have fast ramp-up ability during load pick up; furthermore, grid-connected PV systems can automatically isolate itself from the utility grid when a power outage occurs and then reconnect to the grid in a prompt manner once the grid is recovered to support the black start process [18]. Nevertheless, the application of PVs in black start services is still not mature and there are still several challenges in various aspects including protections, stability, variation of irradiance, voltage and frequency control, operation [19]. For example, there is a limited application 
range for PV systems to provide black start services for the reason that the grid following inverters, which are typically adopted by PV systems, are not considered to be suitable for black start purposes. Additionally, it also requires a complex coordinated communication channels to be constructed for PV systems to energise the distribution network [19].

PV systems normally do not operate as individual BSUs, yet they can cooperate with auxiliary power sources such as energy storage systems. In the literature, a few effective and feasible black start strategies that involve the use of PV are demonstrated. In [20], a model of multi-microgrids including PVs and energy storage systems was illustrated based on the control strategy with three-level structure in accordance with hierarchical control theory and the serial restoration strategy. In [21], a model of PV and energy storage system-based threephase/single-phase multi-microgrids was developed, which apply stand-alone and grid-connected operation strategies. To mitigate the intermittency of PV power generation, the uncertainty of the PV-battery combination as a BSU, and the fluctuation of high-power load unit and PV output power, a stratified optimisation strategy was applied in [22] to ensure the output power of PV-battery within the BSU power requirement along with the State of Charge (SOC) of battery within a reasonable range. Furthermore, a multi-objective optimisation model based on the principle of cluster division was designed in [23] to improve the black start efficiency and stability for the PV-battery system.

\subsection{Hydro Power}

Hydro power plants are considered to be desirable sources to provide black start capability. Except for the geographic location and the confining amount of energy resulted from the permanent water reserve, hydro power plants present the least auxiliary power requirement (only excitation system and valve operations for the turbine) compared with other sources and the turbines can be restarted rapidly [19]. According to [24], the hydro generators need only $0.5-1 \%$ of their rated capacity for black start, while nuclear and gas power plants typically require $7-8 \%$ and $1.5-2 \%$ of their rated capacity, respectively. Furthermore, many hydro power plants can contribute large system inertia, and compared with large steam generators, they have relatively high ramp rates when changing power output, and high tolerance for frequency excursions, which can support the system frequency stability [25]. Therefore, hydro power plants tend to be ideal BSUs. A case study presented in [24] indicates that the governor and exciter control play an important role during hydro generator start-up and cold load pick-up process. Furthermore, a mock drill of black start using a hydro power plant in western regional grid of India was carried out in [26] and an experiment of black start using hydro and gas turbine in South Korean power system was accomplished in [27].

\subsection{Battery Energy Storage System (BESS)}

There is a huge potential for BESS applications in assisting power system restoration after blackouts. BESSs not only can act as BSUs and ancillary power suppliers, but also serve as power loads in the early state of the system process to optimise the distribution of power flows and provide ancillary services
[11]. On top of those roles, the BESSs can also mitigate the negative impacts of RESs' characteristics of high volatility and irregularity, stabilise RESs' fluctuate outputs, provide frequency regulation and spinning reserves, support the islanded operation of the networks, and offer reference signals of voltage and frequency [11] [28]. Despite the various desirable properties that BESSs can offer for black start, there are also problems still to be solved such as their locations, energy reservoir availability, expensive cost for large storage capacity, and short lifespans [11] [19]. Furthermore, due to the rarity of occurrence for a blackout event, it is uncommon and not cost effective to install BESSs in a power network purely for the black start services. The inadequate installed capacity of the BESSs and the situation that insufficient battery energy is stored in BESSs when a blackout happens to occur also concerns [11].

Some literature reviews regarding the BESSs performing as an assistant role with other RESs are already presented in section 3.1 and 3.2. Furthermore, in [11], a multi-objective restoration optimisation model with the aid of BESS was developed. The model considers parameters including system topology, BSUs and NBSUs (Non-Black Start Units), support from BESSs, as well as a control strategy so that the BESSs can effectively follow the signals from system dispatchers while keeping the state-of-charge level of BESSs within an acceptable level. In [29], a laboratory scale microgrid model consisting of PVs, wind energy sources, fuel cells and batteries with a smart strategy was shown to smoothly switchover from islanded operation to grid-connected operation during black start and recovery process.

\subsection{Electric Vehicles $(E V S)$ and $V 2 G$}

In recent years, the demand for EVs has prompted the extensive construction of $\mathrm{EV}$ charger infrastructure. As suggested in [1], it is anticipated that almost $80 \%$ of households will smart charge their EVs and almost $45 \%$ of which actively provide V2G (vehicle-to-grid) services by 2050 in the UK. The EVs can be treated as mobile batteries which are capable of providing power back to the grid (i.e. V2G), however, the EVs cannot act as a reliable BSU due to their uncertain state of charge/discharge in a certain time so that the reserve capacity from EVs suffers [10]. Besides, the low deployment rate of EVs currently also results in poor network energisation capability and low availability. Therefore, these drawbacks above call for advanced communication and control systems to be installed with EVs. In the literature, there are a few explorations of applying EVs in black start, for instance, the potentials of a bi-directional EV charger based emergency power supply system was investigated through a black start procedure in [30], whereas in [31], an intelligent integrated station of EVs was put forward which owns a higher start-up rate, a better efficiency, and a more secure and economical restoration process. 


\section{Case Study}

\subsection{System Description}

The purpose of this case study is to investigate the feasibility of using DERs to provide black start capability, particularly in frequency regulation. The case study in this paper is based on a simulation of an islanded microgrid model in Matlab/Simulink platform. The structure of the islanded microgrid model is shown in Fig. 1, which includes a voltage bus, a Hydraulic Turbine driven Synchronous Generator (HTSG), a wind generator, a BESS and primary load that has the highest priority to connect to the voltage bus. The microgrid is completely isolated from the utility grid which mimics the situation following a blackout, and the model adopts the HTSG as the main power supply. The rated value of the bus voltage is $11 \mathrm{kV}$, and the nominal system frequency is $50 \mathrm{~Hz}$.

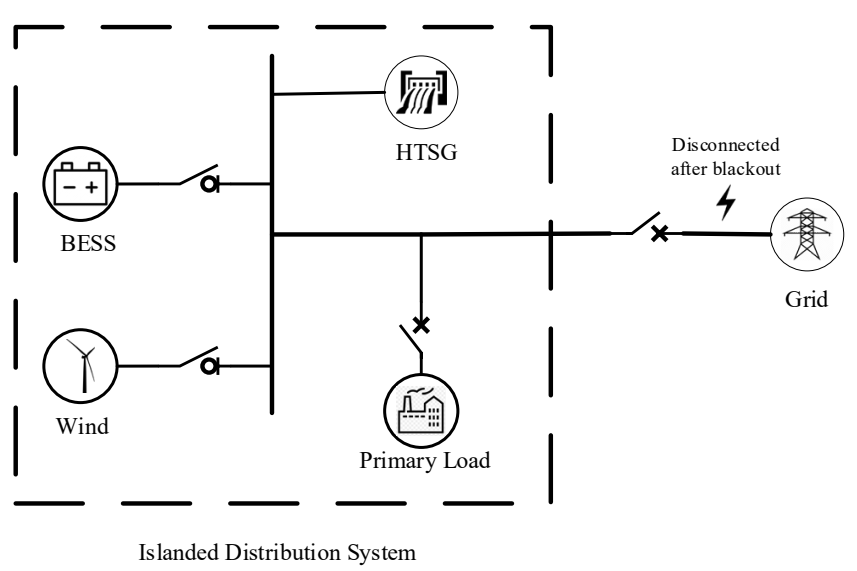

Fig. 1. Connection diagram of the test microgrid

As mentioned in section 3.3, hydro power plants have many properties that are desirable for providing black start services, and it can be widely used in remote islanded microgrids. The HTSG used in this paper is a mini hydro power plant, rated at $5 \mathrm{MW}$. It is also equipped with an excitation system of IEEE standard 421.5 [32] and a hydraulic turbine governor which includes transient droop compensation [33]. This paper also uses two inverters based DERs to emulate a wind generator and a BESS, and both apply the P/Q control. The power ratings of the wind generator and BESS are 0.2 MW and 0.3 MW respectively. The primary load demand is set to be $1.05 \mathrm{MW}$.

\subsection{Black Start Procedure}

The whole black start procedure is divided into two steps: the energising step and the load pick-up step. Once the blackout happens, the microgrid is disconnected from the main grid, and then the HTSG starts to establish the voltage and the frequency within the network. After that, the primary load is ready to connect to the microgrid bus. At the same time, when the DERs detect the frequency sag caused by the load step, they will increase their active power to retain the system frequency. The case study will investigate 3 scenarios based on different DERs combination, namely HTSG only, HTSG + BESS, and HTSG + BESS + Wind. The focus of the case study is on the feasibility of providing black start capability by the coordination of different DERs, especially on frequency regulation. In this case study, the total simulation time is set to be $30 \mathrm{~s}$, and the load pick-up step starts at $10 \mathrm{~s}$.

\subsection{Scenario 1 - HTSG Only}

When considering the scenario where the whole black start process relies only on the HTSG, the frequency can be established and maintained as $50 \mathrm{~Hz}$ in the energising step. However, in the load pick-up step, the frequency drops to around $45 \mathrm{~Hz}$, which is shown as the blue curve in Fig. 5. According to EREC G59 [34], when the system frequency falls below $47.5 \mathrm{~Hz}$ for $20 \mathrm{~s}$ or below $47 \mathrm{~Hz}$, the DERs will be disconnected and the black start process would fail. Furthermore, different load size integrations can result in different degrees of frequency disturbance. As it is shown in Fig. 2, there is an unexpected drop in frequency as the load is picked up, and the larger size the load is connected, the more significant degree the frequency is disturbed. Besides, once the frequency is decreased to a certain level, it takes a very long time for the frequency to recover. This unexpected phenomenon is caused due to the characteristics of the hydraulic turbine governor of the HTSG and it can be harmful to power system stability. Therefore, it is necessary to utilise additional DERs to perform as auxiliary support to the mini hydro power plant during the black start process.

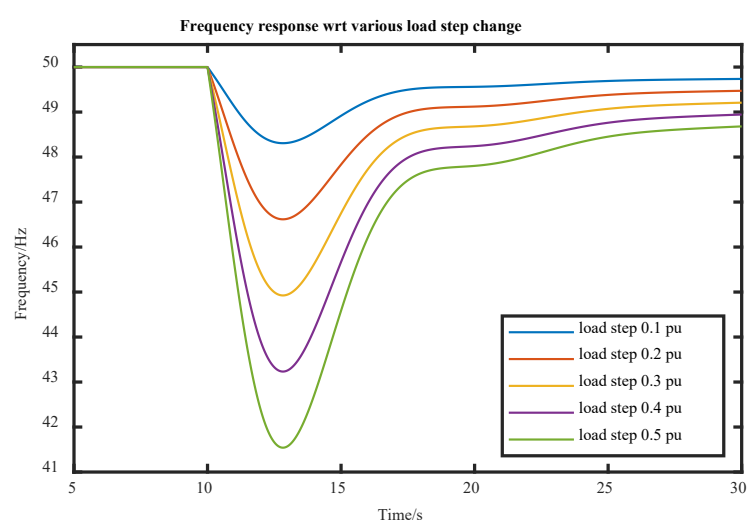

Fig. 2. Frequency response against various load step change

\subsection{Scenario $2-H T S G+B E S S$}

When the HTSG established the voltage and the frequency on the bus after the blackout, the BESS starts to generate power output when the load step is detected. The BESS can react to the load step in $0.1 \mathrm{~s}$. Besides, the BESS also owns a very high ramp rate, normally delivering full output power within $0.2 \mathrm{~s}$ [35].

The power output is shown in Fig. 3 and the frequency variation is shown as the red curve in Fig. 5. It can be seen that, compared to scenario 1 , the frequency drops to around $46 \mathrm{~Hz}$ rather than around $45 \mathrm{~Hz}$ when the load is picked up. In terms of the power output, it can be clearly seen that the BESS is able to increase its power output instantly and rapidly as expected. The results can validate that it is feasible to use an additional BESS to assist the black start process and the cooperation of the DERs can improve black-start performance. 


\subsection{Scenario $3-H T S G+$ BESS + Wind}

Although the frequency is improved considerably in scenario 2 , the frequency is still outside the acceptable range (above 47 $\mathrm{Hz}$ ). Hence, a wind generator is applied as another auxiliary support for black start. The wind generator also owns a rapid ramping-up capability, and in this case study, the wind speed is assumed to be constant during the entire black start process so as to keep the wind power output in a stable manner.

The power output is shown in Fig. 4 and the frequency variation is shown as the yellow curve in Fig. 5. It can be seen that the frequency drops to approximately $47 \mathrm{~Hz}$ at $10 \mathrm{~s}$ and then recover to $50 \mathrm{~Hz}$ speedily. The results can validate that wind turbine is also feasible to be utilised as a BSU in the black start process.

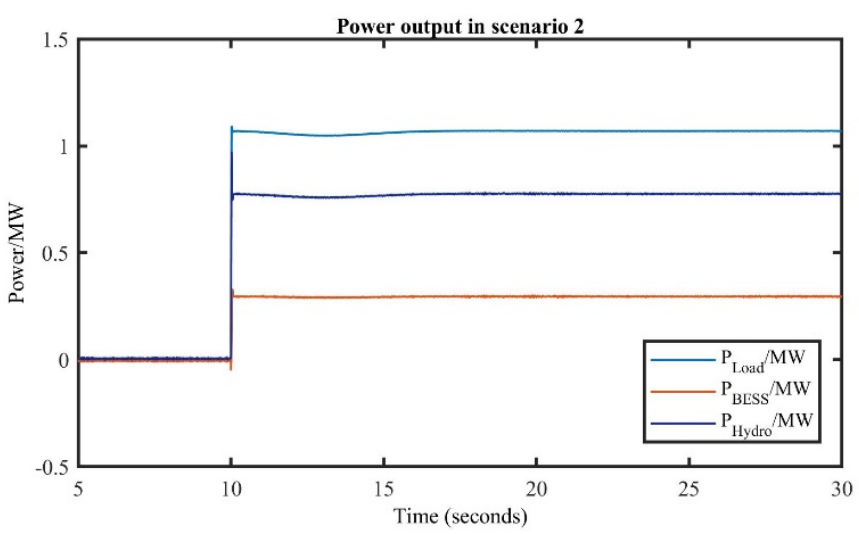

Fig. 3. Power output in scenario 2

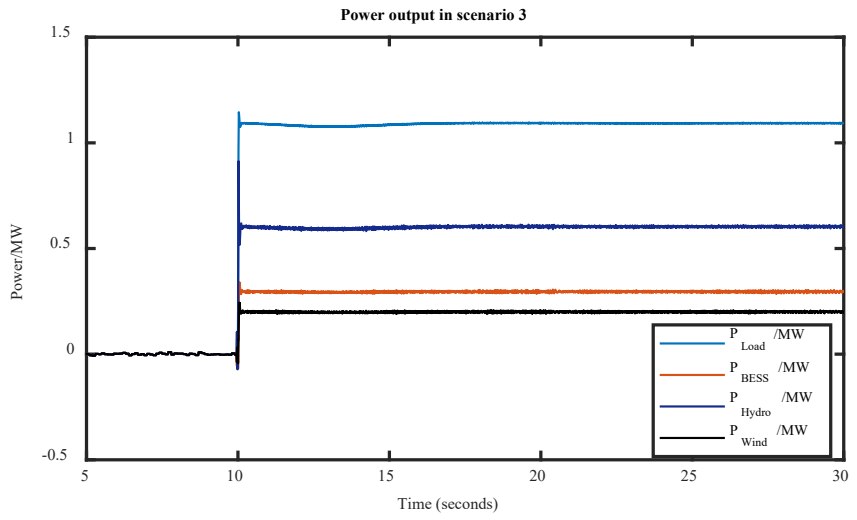

Fig. 4. Power output in scenario 3

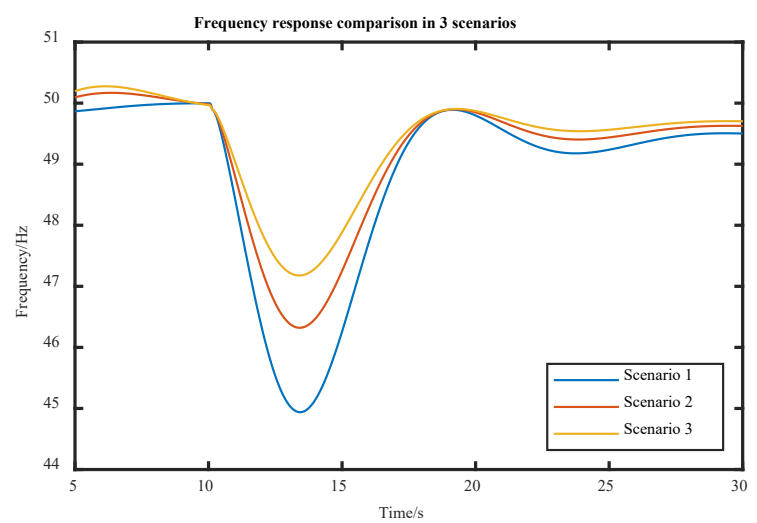

Fig. 5. Frequency response comparison in scenarios 1, 2 and 3

The three scenarios outlined above can be used to demonstrate the feasibility of using DERs to provide black-start capability, particularly in terms of frequency regulation during black start. As demonstrated in the case study, the frequency can be significantly improved by increasing the auxiliary power support, which results in a higher frequency nadir and a less oscillating final steady state. However, increased auxiliary power support does not necessarily imply improved performance; in fact, it may result in frequency overshoot. As a result, it is critical to have an optimised auxiliary power sizing from DERs so that the response can be coordinated optimally among the available resources. Additionally, different types of generators respond differently to demand changes and they are capable of ramping up quickly in response to load changes, which validates the black start capability of DERs discussed in Section 3.

Other aspects including voltage control, transformer energisation, and other renewables integration (e.g. PV) also need further investigation. Furthermore, considering several restrictions, including the SOC of BESS when blackout occurs, the intermittent power output from wind turbines due to its natural constraints, the coordination among different DERs, and variation of load demand, the power outputs and the frequency response may not be shown as ideal as aforementioned. Therefore, further investigations need to be conducted, e.g. BESS SOC management, communication and control methods among multiple DERs, optimal allocation of various DERs, load change/DER output forecast.

\section{Conclusion}

This paper explores the feasibility of utilising DERs to provide a "bottom-up" black start approach to replace the conventional "top-down" approach in the context of decommissioning of traditional thermal power plant and expanding the utilisation of the RESs in modern power system. Several aspects regarding black start studies using DERs are reviewed, including technical requirements for DERs in black start procedure, and suitability of applications of various types of DERs in black start. A series of case studies based on a microgrid model have also been performed proving that it is feasible to use a BESS and a wind generator to act as auxiliary support to a mini hydro power plant to provide an adequate black start capability rather than purely relying on one hydro 
plant. Additionally, further considerations and future research are discussed.

\section{References}

[1] NationalGrid, "Future Energy Scenarios," 2020.

[2] HMGovernment, "The Clean Growth Strategy - Leading the way to a low carbon future," 2017.

[3] W. Yan, D. Tzelepis, and A. Dyśko, "Islanding of Distribution Networks: Challenges and Potential Solutions," in 2018 53rd International Universities Power Engineering Conference (UPEC), 4-7 Sept. 2018 2018, pp. 1-6.

[4] K. Sun, Y. Hou, W. Sun, and J. Qi, Power System Control Under Cascading Failures: Understanding, Mitigation, and System Restoration. Wiley-IEEE Press, 2019.

[5] NationalGrid, "System Operability Framework - Black start from distributed sources," 2017.

[6] C. Gouveia, C. Moreira, A. G. Madureira, J. Gouveia, D. Issicaba, and J. A. P. Lopes, "Black start and islanding operations of microgrid," in Power and Energy, Variability, Scalability and Stability of Microgrids: Institution of Engineering and Technology, 2019, pp. 463-495.

[7] W. Sun, C. Liu, and S. Liu, "Black start capability assessment in power system restoration," in 2011 IEEE Power and Energy Society General Meeting, 24-28 July 20112011 , pp. 1-7.

[8] J. W. Feltes and C. Grande-Moran, "Black start studies for system restoration," in 2008 IEEE Power and Energy Society General Meeting - Conversion and Delivery of Electrical Energy in the 21st Century, 20-24 July 2008 2008, pp. 1-8.

[9] MottMacDonald, "Black Start Alternative Approaches," 2015.

[10] NationalGrid, "Black Start from Non-Traditional Generation Technologies - Technology capabiliy and readiness for distributed restoration," 2019.

[11] W. Liu, L. Sun, Z. Lin, F. Wen, and Y. Xue, "Multiobjective restoration optimisation of power systems with battery energy storage systems," IET Generation, Transmission \&amp; Distribution, vol. 10, no. 7, pp. 1749-1757.

[12] M. Aktarujjaman, M. A. Kashem, M. Negnevitsky, and G. Ledwich, "Black start with dfig based distributed generation after major emergencies," in 2006 International Conference on Power Electronic, Drives and Energy Systems, 12-15 Dec. 2006 2006, pp. 1-6.

[13] T. Wei, W. Hongtao, and J. Yingzhe, "Construction and control strategy research of black start unit containing wind farm," in TENCON 2015 - 2015 IEEE Region 10 Conference, 1-4 Nov. 2015 2015, pp. 1-5.

[14] A. Golshani, W. Sun, Q. Zhou, Q. P. Zheng, and Y. Hou, "Incorporating Wind Energy in Power System Restoration Planning," IEEE Transactions on Smart Grid, vol. 10, no. 1, pp. 16-28, 2019.

[15] D. Jianfeng, T. Yi, W. Qi, D. Xianbo, L. Chenlong, and F. Lixin, "Black start technology for local power grid via PMSG-based wind power generation," in 2017 IEEE 3rd International Future Energy Electronics Conference and
ECCE Asia (IFEEC 2017 - ECCE Asia), 3-7 June 2017 2017, pp. 2025-2030.

[16] L. Liu, J. Wu, Z. Mi, and C. Sun, "A feasibility study of applying storage-based wind farm as black-start power source in local power grid," in 2016 International Conference on Smart Grid and Clean Energy Technologies (ICSGCE), 19-22 Oct. 2016 2016, pp. 257 261.

[17] D. Jie and R. G. Harley, "Islanded microgrids black start procedures with wind power integration," in 2013 IEEE Power \& Energy Society General Meeting, 21-25 July 2013 2013, pp. 1-5.

[18] A. M. El-Zonkoly, "Renewable energy sources for complete optimal power system black-start restoration," IET Generation, Transmission \& Distribution, vol. 9, no. 6, pp. 531-539, 2015.

[19] ELIA, "Study on the Review of the Black Start Ancillary Services," 2018.

[20] L. Yu et al., "Study on black start strategy of multimicrogrids with PV and energy storage systems considering general situations," in 2015 6th International Conference on Power Electronics Systems and Applications (PESA), 15-17 Dec. 2015 2015, pp. 1-6.

[21] Z. Xu, P. Yang, Z. Zeng, Y. Zhang, J. Peng, and Q. Zheng, "Study on black start strategy for multi-microgrids," in 2016 IEEE Innovative Smart Grid Technologies - Asia (ISGT-Asia), 28 Nov.-1 Dec. 2016 2016, pp. 144-148.

[22] J. Li, H. You, J. Qi, M. Kong, S. Zhang, and H. Zhang, "Stratified Optimization Strategy Used for Restoration With Photovoltaic-Battery Energy Storage Systems as Black-Start Resources," IEEE Access, vol. 7, pp. 127339127352, 2019.

[23] L. Tongge, H. Yonghong, M. Junyi, and X. Yimin, "The Research on Black Start Strategy of Distributed Photovoltaic-Battery Energy Storage Systems Based on Cluster Division," in 2020 IEEE Sustainable Power and Energy Conference (iSPEC), 23-25 Nov. 2020 2020, pp. 337-342.

[24] S. R. Kurup and S. Ashok, "Performance of a hydro power plant during black start and islanded operation," in 2015 IEEE International Conference on Signal Processing, Informatics, Communication and Energy Systems (SPICES), 19-21 Feb. 2015 2015, pp. 1-5.

[25] J. Gracia, L. Markel, D. Rizy, P. O'Connor, R. Shan, and A. Tarditi, "Hydropower Plants as Black Start Resources," 2019.

[26] P. Mukhopadhyay et al., "Black start experiences for 400 $\mathrm{kV}$ hydro power plant in western regional grid of India," in 2016 National Power Systems Conference (NPSC), 1921 Dec. 2016 2016, pp. 1-6.

[27] S. Lee, "Hydro and gas turbine blackout restoration strategies in the South Korean power system," in 2014 IEEE PES General Meeting | Conference \& Exposition, 27-31 July 2014 2014, pp. 1-6.

[28] A. D. Giorgio, A. Giuseppi, F. Liberati, and A. Pietrabissa, "Controlled electricity distribution network black start with energy storage system support," in 2017 25th Mediterranean Conference on Control and Automation (MED), 3-6 July 2017 2017, pp. 781-786. 
[29] S. Thale and V. Agarwal, "A smart control strategy for the black start of a microgrid based on PV and other auxiliary sources under islanded condition," in 2011 37th IEEE Photovoltaic Specialists Conference, 19-24 June 2011 2011, pp. 002454-002459.

[30] Y. Song, P. Li, Y. Zhao, and S. Lu, "Design and Integration of the Bi-directional Electric Vehicle Charger into the Microgrid as Emergency Power Supply," in 2018 International Power Electronics Conference (IPECNiigata 2018 -ECCE Asia), 20-24 May 2018 2018, pp. 3698-3704.

[31] J. Sun, D. Xie, Y. Lou, M. Yang, and Y. Zhang, "Blackstart scheme based on EV's intelligent integrated station," in 2014 International Conference on Power System Technology, 20-22 Oct. 2014 2014, pp. 3118-3123.

[32] I. 421.5-1992, "Recommended Practice for Excitation System Models for Power System Stability Studies," 1992.

[33] P. Kundur, Power system stability and control. New York: New York : McGraw-Hill, 1998.

[34] ENA, "Engineering Recommendation G59 Recommendations for the Connection of Generating Plant to the Distribution Systems of Licensed Distribution Network Operators," 2015.

[35] NationalGrid, "Black Start from Non-Traditional Generation Technologies - Power Island Strength and Stability in support of Black Start," 2019. 